\title{
DRIPPING AND JETTING IN COFLOWING LIQUID STREAMS
}

\author{
SIU-LONG LEI*,†,‡ and XIAOPING WANG ${ }^{\dagger, \S}$ \\ *Department of Mathematics, \\ University of Macau, \\ Macau, China \\ ${ }^{\dagger}$ Department of Mathematics, \\ The Hong Kong University of Science and Technology, \\ Clear Water Bay, Kowloon, Hong Kong, China

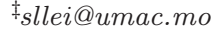 \\ §mawang@ust.hk
}

\begin{abstract}
Drop formation and dripping and jetting phenomena occur in an extremely large variety of situation, spanning a broad range of physical length scales. In this paper, we study the dynamics of dripping-to-jetting transition for two immiscible coflowing liquid streams numerically. Two different classes of transition are identified. In both cases, nonlinear dynamical phenomena such as period doubling and chaos are observed between simple dripping and jetting. Extensive numerical calculations show that the first class of dripping-to-jetting transition is determined by the Weber number of the inner fluid $\mathcal{W}_{\text {in }}$, and the second class of dripping-to-jetting transition is controlled by capillary number of the outer fluid $\mathcal{C}_{\text {out }}$.
\end{abstract}

Keywords: Dripping jetting transition; phase field simulations.

\section{Introduction}

A liquid forced through an orifice will ultimately break into drops through one of two mechanisms. At slow flows, the emerging liquid drips from the orifice, whereas at faster flows, the liquid forms a thin stream that breaks into drops away from the orifice; these are the dripping and jetting regimes. This behavior is familiar to anyone who has slowly increased the flow rate of water at a kitchen faucet. The dripping-to-jetting transition is sharp if the liquid viscosity is large compared to water [Ambravaneswaran et al. (2004)], whereas the transition initially becomes chaotic [Clanet and Lasheras (1999); Shaw (1984); Ambravaneswaran et al. (2000); Coullet et al. (2005)] before jetting for lower viscosity fluids. The jets eventually break into drops due to the Rayleigh-Plateau instability [Plateau (1849); Rayleigh (1879)]. In fact, for lower viscosity fluid, complex dripping such as period doubling, chaos, and hysteresis can be observed between simple dripping and jetting.

Here, we consider a liquid is injected in a second immiscible liquid and they are coflowing with different velocities. Dripping occurs at low flow rates of both fluids 
and is characterized by the periodic formation of individual drops that pinch-off from the tip. For jetting, Utada et al. [2007] show, through physical experiments, that there are two different classes of jetting. One is driven by the flow rate of the inner fluid and the other is driven by the flow rate of the outer fluid. They also show that the two classes of dripping-to-jetting transition are controlled by the Weber number of the inner fluid $\mathcal{W}_{\text {in }}$ and the capillary number of the outer fluid $\mathcal{C}_{\text {out }}$.

In this paper, the problem is studied numerically by using the phase-field model with appropriate boundary conditions. Dripping and jetting are simulated numerically and the two classes of dripping-to-jetting transition observed in [Utada et al. (2007)] are identified. It was shown that the transition is controlled by the Weber number of the inner fluid $\mathcal{W}_{\text {in }}$ and the capillary number of the outer fluid $\mathcal{C}_{\text {out }}$. Besides, nonlinear dynamical phenomena such as period doubling and chaos are observed.

\section{Mathematical Model}

Phase field models have been developed and analyzed for the two phase fluid flow by many researchers, see e.g., [Hohenberg and Halperin (1977); Anderson et al. (1998)]. More recently, a phase field model together with the generalized Navier boundary condition is proposed to study the moving contact line problem [Qian et al. (2003); Wang et al. (2008)]. This model is employed here to model the dripping-to-jetting transition of coflowing liquid streams in 2D Cartesian coordinates with constant fluid density $\rho$ and constant fluid viscosities $\eta$. The two coupled equations of motion are the Cahn-Hilliard Eq. (1) for the phase field $\phi(\mathbf{r})$ and the NavierStokes Eq. (2) in the presence of the capillary force. The boundary condition for $\phi(\mathbf{r})$ is the dynamic relaxational Eq. (3) at solid surface and the boundary condition for Navier-Stokes equation is the GNBC (4), governing the fluid slip at solid surface. In dimensionless form, the system can be written as

$$
\begin{gathered}
\frac{\partial \phi}{\partial t}+\mathbf{v} \cdot \nabla \phi=\mathcal{L}_{d} \nabla^{2}\left(-\nabla^{2} \phi-\phi+\phi^{3}\right), \\
\mathcal{R}\left[\frac{\partial \mathbf{v}}{\partial t}+(\mathbf{v} \cdot \nabla) \mathbf{v}\right]=-\nabla p+\nabla^{2} \mathbf{v}+\mathcal{B}\left(-\nabla^{2} \phi-\phi+\phi^{3}\right) \nabla \phi \\
\frac{\partial \phi}{\partial t}+v_{x} \partial_{x} \phi=-\mathcal{V}_{s}\left[\partial_{n} \phi-\frac{\sqrt{2}}{3} \cos \theta_{s} s_{\gamma}(\phi)\right], \\
\mathcal{L}_{s}^{-1} v_{x}^{\text {slip }}=-\partial_{n} v_{x}+\mathcal{B}\left[\partial_{n} \phi-\frac{\sqrt{2}}{3} \cos \theta_{s} s_{\gamma}(\phi)\right] \partial_{x} \phi,
\end{gathered}
$$

with $s_{\gamma}(\phi)=(\pi / 2) \cos (\pi \phi / 2)$. Here, six dimensionless parameters appear in the model: (1) $\mathcal{L}_{d}=M r / \xi V$, which is the ratio of the diffusion length $M r / V$ to $\xi$, (2) $\mathcal{R}=\rho \xi V / \eta$, (3) $\mathcal{B}=r^{2} \xi / u \eta V=\frac{3}{2 \sqrt{2}} \gamma / \eta V$, which is inversely proportional to the capillary number $C a=\eta V / \gamma$, (4) $\mathcal{V}_{s}=K \Gamma / V$, which is the ratio of $K \Gamma$ (of velocity dimension) to $V$, (5) $\mathcal{L}_{s}=l_{s} / \xi$, which is the ratio of the slip length to $\xi$, and (6) the 
static contact angle $\theta_{s}$. In our calculations, the values of the first four parameters are taken from those determined through MD simulations of moving contact line problem [Qian et al. (2003)] (used also in [Qian et al. (2009); Wang et al. (2008); Wu et al. (2010)]). They are $\mathcal{L}_{d}=5, \mathcal{R}=0.03, \mathcal{B}=12$, and $\mathcal{V}_{s}=5$, with the length scale $\xi=1 / 3 \sigma$ and the velocity scale $V=0.25 \sqrt{\epsilon / m}$ (in which $\epsilon$ and $\sigma$ are energy and length scales, respectively in the Lennard-Jones potential for fluid molecules and $m$ is the fluid molecular mass). We use the above values of $\xi$ and $V$ as the length and velocity units. The static contact angle $\theta_{\mathrm{s}}$ is taken to be $180^{\circ}$ so that the top and bottom solid boundary does not like the inner fluid completely and we take $\mathcal{L}_{\mathrm{s}}=10.1$. Note that, from physical point of view, the static contact angle and the slip length have less effect on the dynamics of dripping and jetting of coflowing liquid streams. Numerically, the result in Figs. 4-6 can be reproduced by changing the static contact angle $\theta_{\mathrm{s}}$ to $110^{\circ}$.

The system (1)-(4) is considered on the rectangular domain

$$
\Omega=\{(x, z) \mid 0 \leq x \leq L, 0 \leq z \leq H\} .
$$

The orifice for the entrance of the inner fluid is located in the middle of the left boundary and the width of the orifice is denoted by $H_{\text {orifice }}$.

On the top and bottom boundary, the boundary condition for $\phi$ is given by the dynamic relaxational Eq. (3) and the boundary condition for the velocity field $\mathbf{v}$ is the GNBC (4). On the left boundary, $\phi$ is given by the piecewise function

$$
\phi(z)= \begin{cases}-1, & \text { if }\left|z-\frac{1}{2} H\right| \leq \frac{1}{2} H_{\text {orifice }} \\ 1, & \text { elsewhere }\end{cases}
$$

This step function specifies that the red fluid is inside the orifice and blue fluid is outside the orifice. For the velocity field, $v_{z}(z)=0$ and $v_{x}(z)$ is also a piecewise function on the left boundary. In fact, suppose $Q(z)$ is the Poiseuille-type quadratic profile with average velocity $U$, satisfying $-\partial_{x} p+\partial_{z}^{2} v_{x}=0$ with $\int_{0}^{H} d z v_{x}(z)=U H$, and is consistent with the slip boundary conditions at the top and bottom surfaces. Then

$$
v_{x}(z)= \begin{cases}u_{\mathrm{in}}, & \text { if }\left|z-\frac{1}{2} H\right| \leq \frac{1}{2} H_{\text {orifice }} \\ Q(z), & \text { elsewhere }\end{cases}
$$

where $u_{\text {in }}$ is the average velocity of the inner fluid. Therefore, the flow rate of the inner fluid $q_{\text {in }}$ is given by $u_{\text {in }} H_{\text {orifice }}$ and the flow rate of the outer fluid is

$$
q_{\text {out }}=U H-\int_{\left|z-\frac{1}{2} H\right| \leq \frac{1}{2} H_{\text {orifice }}} d z Q(z)=2 \int_{0}^{\frac{1}{2}\left(H-H_{\text {orifice }}\right)} d z Q(z) .
$$

Then, the average velocity of the outer fluid is $u_{\text {out }}=q_{\text {out }} /\left(H-H_{\text {orifice }}\right)$. Note that, $U \neq u_{\text {out }}$, but $U$ can characterize $u_{\text {out }}$. Therefore, in the following computations, 
$U$, which is a input parameter in our computer code, is used to replace $u_{\text {out }}$. On the right boundary, $v_{z}=0, v_{x}=Q$ (the Poiseuille quadratic profile) and Neumann boundary condition is used for $\phi$, i.e., $\partial_{x} \phi=0$.

\section{Numerical Results}

In this section, we study the dynamics in the dripping-to-jetting transition by solving our mathematical model numerically on a rectangular domain with $L=200$, $H=40$, and $H_{\text {orifice }}=4.375$. We use a pressure-Poisson solver [Johnston and Liu (2002)] for the Navier-Stokes equation and the first-order Euler method for the diffusion equation. The discretization of the continuum model is based on finite differences and the key steps are detailed and explained in the appendix of [Qian et al. (2003)]. The PDE system is solved on a 512-by-64 rectangular mesh with time step $\Delta t=2.67 \times 10^{-4}$. The initial condition for the phase field is $\phi \equiv 1$, i.e., there is red fluid only in the beginning, and the initial conditions for the velocity field are $v_{z} \equiv 0$ and $v_{x}$ is the same as the left boundary condition, i.e., $v_{x}(x, z)=v_{x}(0, z)$ for all $x$.

\subsection{Two classes of dripping-to-jetting transition}

For a liquid is injected in a second immiscible liquid and coflowing with different flow rates, dripping occurs at low flow rates of both fluids and is characterized by the periodic formation of individual drops that pinch-off from the tip. Figure 1 shows a numerical simulation of dripping. In this simulation, we take $u_{\text {in }}=1.5$ and $U=3$.

For jetting, in [Utada et al. (2007)], two distinct classes of transition from dripping to jetting are observed from physical experiments. The first is driven by the flow rate of the inner fluid; as it is increased, the dripping drop is pushed downstream and is ultimately pinched off at the end of the resultant jet, see Fig. 2 for a numerical simulation of the first class jetting. In the simulation, we take $u_{\text {in }}=3.2$ and $U=3$.

The second class of transition is driven by the flow rate of the outer fluid; as it is increased, drops formed at the tip decrease in size until a jet is formed, whereupon drop breakup occurs downstream at the end of the thin jet, see Fig. 3 for a numerical simulation of the second class jetting. In the simulation, we take $u_{\text {in }}=1.9$ and $U=4.5$.

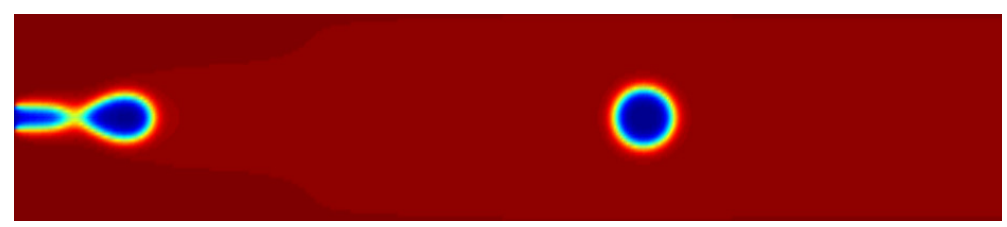

Fig. 1. Dripping: $u_{\text {in }}=1.5$ and $U=3$. 


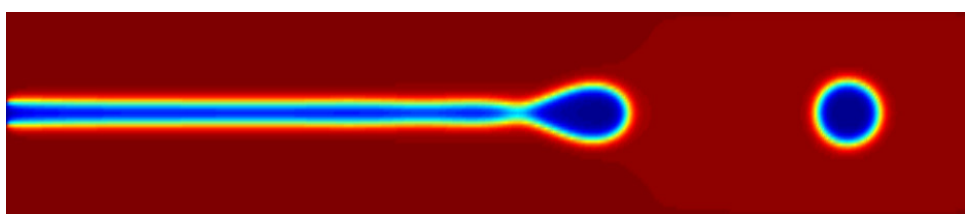

Fig. 2. The first class jetting: $u_{\text {in }}=3.2$ and $U=3$.

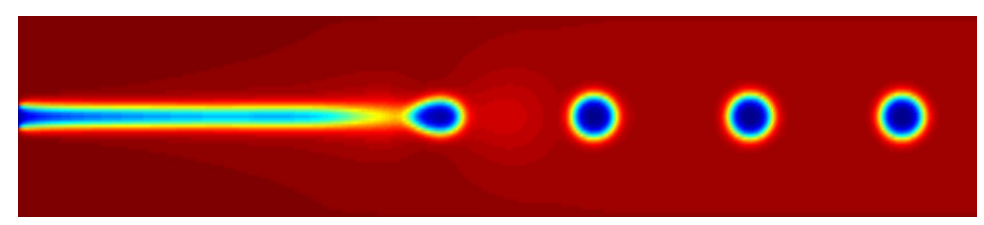

Fig. 3. The second class jetting: $u_{\text {in }}=1.9$ and $U=4.5$.

\subsection{Nonlinear dynamical phenomena}

From numerical experiments, we measure the time interval between droplets, $t_{1}, t_{2}, \ldots, t_{i}, \ldots$, where $t_{i}$ is the time interval between the $i$ th and $(i+1)$-th droplet. Time interval data are then examined through time return maps, where each point in the map is determine by the ordered pair $\left(t_{n}, t_{n+1}\right)$ for some $n$, to make inferences about the nonlinear dynamics of $\left\{t_{n}\right\}$. A response is called period- $k$ if there exists a positive integer $N$ such that $t_{n+k}=t_{n}$ for all $n \geq N$. In the following, we consider the two different classes of dripping-to-jetting transitions identified in Sec. 3.1.

For the first class of dripping-to-jetting transition, we compute the numerical solutions of the Cahn-Hilliard-Navier-Stokes model for $U=3$ and different values of $u_{\text {in }}(1.5-2.5)$. For $u_{\text {in }}=1.5$ to 1.945 , the dripping is simple and period-1, i.e., $t_{n}$ is a constant for $n$ is large enough. Figure 4 (top) shows the time return map for $u_{\text {in }}=1.9$ and the inset shows the sequence $\left\{t_{n}\right\}$ for some $n$. It is found that $\left\{t_{n}\right\}$ is decreasing in the beginning and then keep constant for $n \geq 6$. Therefore, it is a period-1 response and so that the order pair $\left(t_{n}, t_{n+1}\right)$ represent the same point for all $n \geq 6$ on the diagonal (blue line) of the time return map. Hence, there is only one point on the time return map. Figure 4 (bottom) shows the contour $\phi=0$ for the 22nd to 25 th pinch-off. It can be seen that the distance between all droplets are the same.

For $u_{\text {in }}$ is larger than 1.945, the dripping changes to be complex and nonlinear dynamical phenomena such as bifurcation and chaos are observed. Figure 5 (top) shows the time return map for $u_{\text {in }}=1.95$ and the inset shows the sequence $\left\{t_{n}\right\}$ for some $n$. It is found that $t_{n}=t_{n+2}$ for $n \geq 50$ so that the response is of period- 2 . The order pair $\left(t_{n}, t_{n+1}\right)$ represent two different points symmetric about the diagonal of the time return map for all $n \geq 50$. Figure 5 (bottom) shows the contour of $\phi=0$ for the 59 th to $62 \mathrm{nd}$ pinch-off. Note that there are two different distances between consecutive two droplets. 

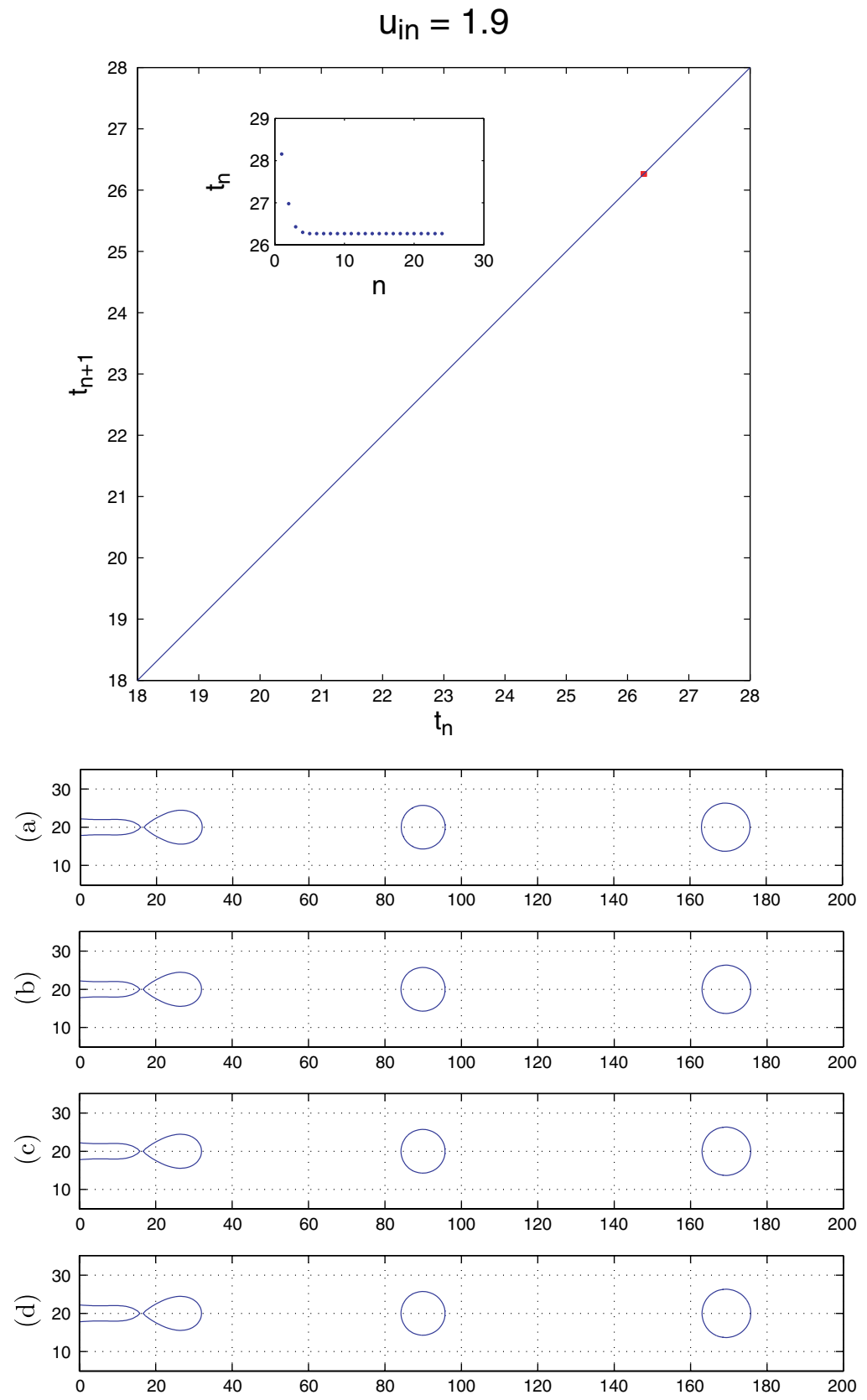

Fig. 4. Period-1 response. Top: time return maps for $U=3$ and $u_{\text {in }}=1.9$. Inset: $t_{n}$ versus $n$. Bottom: zero-contour of $\phi$ : (a) droplet \#22; (b) droplet \#23; (c) droplet \#24; and (d) droplet \#25. 

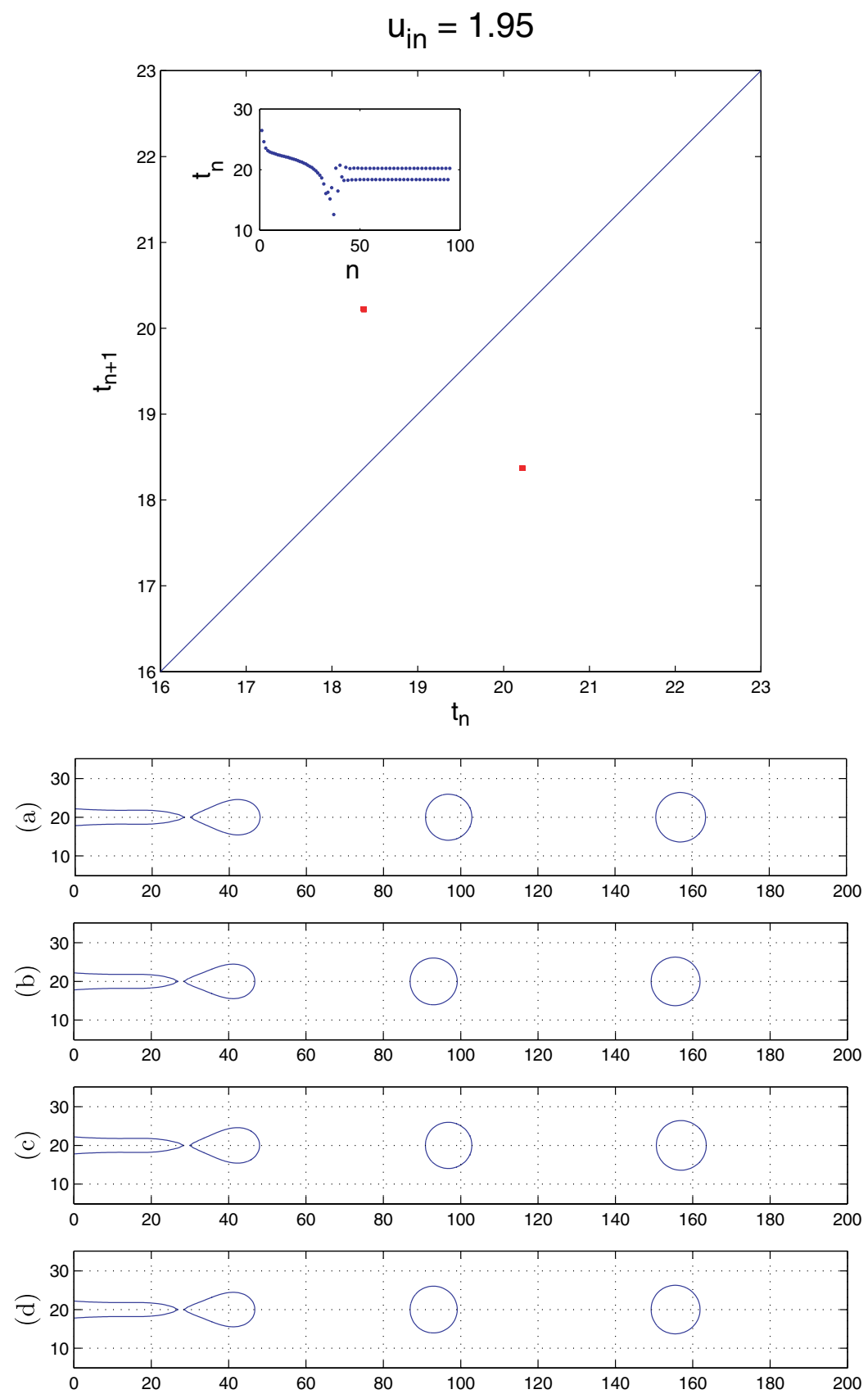

Fig. 5. Period-2 response. Top: time return maps for $U=3$ and $u_{\text {in }}=1.95$. Inset: $t_{n}$ versus $n$. Bottom: zero-contour of $\phi$ : (a) droplet \#59; (b) droplet \#60; (c) droplet \#61; and (d) droplet \#62. 
Figure 6 (top) shows the time return map for $u_{\text {in }}=1.97$ and the inset shows the sequence $\left\{t_{n}\right\}$ for some $n$. It is found that $t_{n}=t_{n+4}$ for $n \geq 40$ so that the response is of period-4. The order pair $\left(t_{n}, t_{n+1}\right)$ represent four different points on the time return map for all $n \geq 40$. Figure 6 (bottom) shows the contour of $\phi=0$ for the 59th to 62 nd pinch-off. Note that there are four different distances between consecutive two droplets and two of them ((a) and (c)) are almost the same. Figure 7 shows the time return maps for $u_{\text {in }}=1.98$ (top) and $u_{\text {in }}=2.1$ (bottom) correspond to period-8 and chaotic responses, respectively.

The nonlinear dynamical results for the first class of dripping-to-jetting transition are summarized in Table 1 and a bifurcation diagram for periodic $t_{n}$ is shown in Fig. 8. The critical velocity $u_{\text {in }}^{*}$ for the dripping-to-jetting transition is discussed in Sec. 3.3.

For the second class of dripping-to-jetting transition, we compute the numerical solutions of the Cahn-Hilliard-Navier-Stokes model for $u_{\text {in }}=1.9$ and different values of $U(3.05-4.3)$. The nonlinear dynamical results are summarized in Table 2 and there is no period doubling in this case. However, multiperiodic complex dripping can be observed for the second class of dripping-to-jetting transition if the viscosity $\eta$ of both fluids is decreased by $10 \%$, see Fig. 14 in Sec. 3.3. The critical velocity $U^{*}$ for the transition of dripping-to-jetting is discussed in Sec. 3.3.

\subsection{Weber number $\mathcal{W}_{\text {in }}$ and capillary number $\mathcal{C}_{\text {out }}$}

The Weber number of the inner fluid is a dimensionless physical constant defined as $\mathcal{W}_{\text {in }}=\rho_{\text {in }} \bar{H}_{\text {orifice }} \bar{u}_{\text {in }}^{2} / \gamma$ where $\rho_{\text {in }}$ is the density of the inner fluid, $\bar{H}_{\text {orifice }}$ is the width of the orifice and $\bar{H}_{\text {orifice }}=H_{\text {orifice }} \xi$ ( $\xi$ is the length scale), $\bar{u}_{\text {in }}$ is the uniform velocity of the inner fluid and $\bar{u}_{\text {in }}=u_{\text {in }} V$ ( $V$ is the length scale), and $\gamma$ is the interfacial tension. In the mathematical model here, the density of both fluids is the same and $\rho$ is used instead of $\rho_{\text {in }}$ in the definition of $\mathcal{W}_{\text {in }}$. From physics, the Weber number of the inner fluid $\mathcal{W}_{\text {in }}$ tells the balance between interface tension and the inertial forces of the inner fluid. When $\mathcal{W}_{\text {in }}$ is small, interfacial tension dominates and forcing the system to drip. By contrast, when $\mathcal{W}_{\text {in }}$ is large, the inertial forces are large enough to overcome interfacial tension, leading to jetting.

The capillary number of the outer fluid is a dimensionless physical constant defined as $\mathcal{C}_{\text {out }}=\eta_{\text {out }} \bar{u}_{\text {out }} / \gamma$ where $\eta_{\text {out }}$ is the viscosity of the outer fluid, $\bar{u}_{\text {out }}$ is the average velocity of the outer fluid, and $\gamma$ is the interfacial tension. In the mathematical model here, the viscosity of both fluids are the same and $\eta$ is used instead of $\eta_{\text {out }}$, and $\bar{U}=U V$ is used instead of $\bar{u}_{\text {out }}$ in the definition of $\mathcal{C}_{\text {out }}$. From physics, the capillary number of the outer fluid $\mathcal{C}_{\text {out }}$ tells the balance between interfacial tension and the viscous shear stresses on the droplet. When $\mathcal{C}_{\text {out }}$ is small, interfacial tension dominates, forcing the system to drip. By contrast, when $\mathcal{C}_{\text {out }}$ is large, the shear stress on the droplet is large enough to overcome interfacial tension, leading to jetting. 

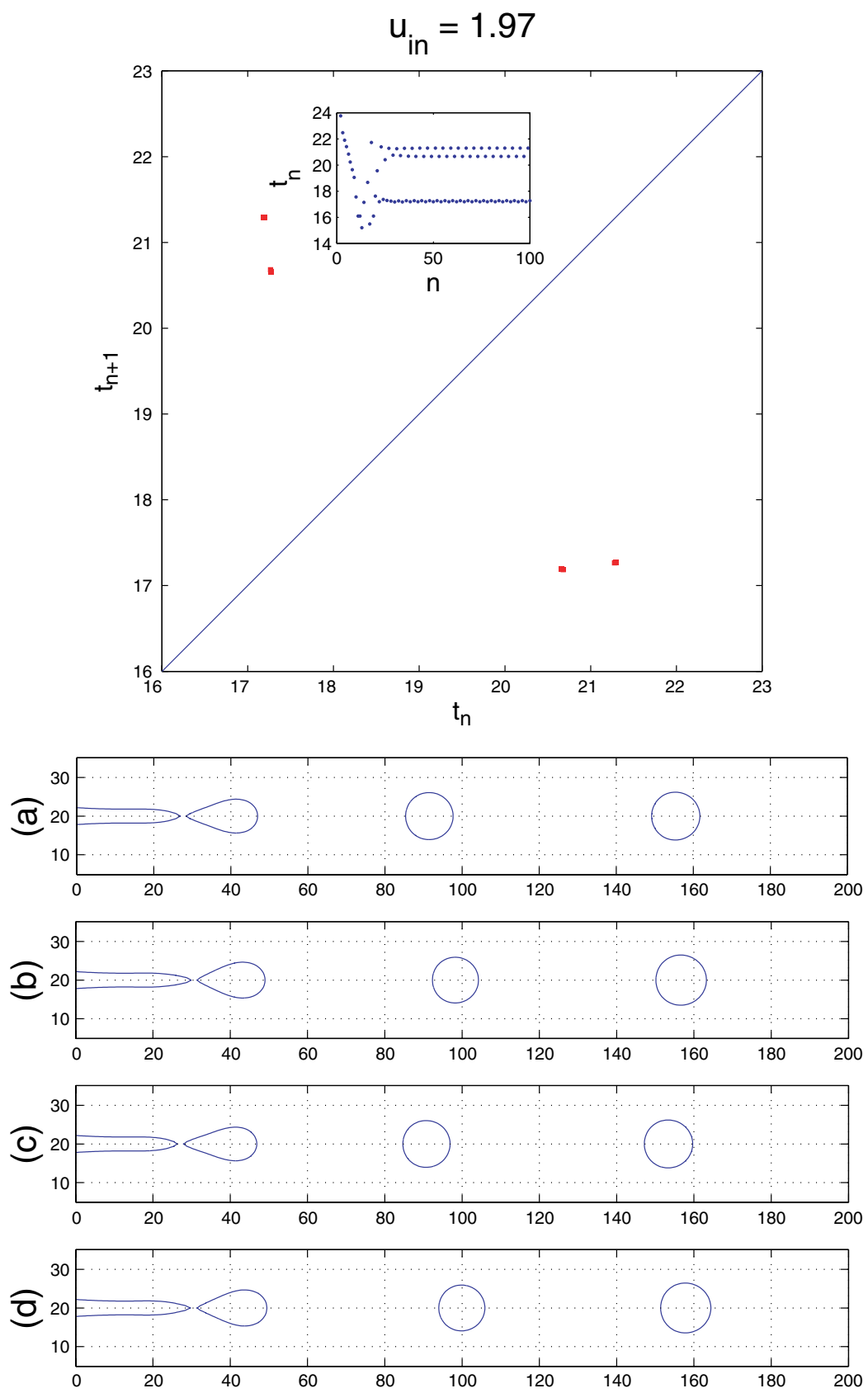

Fig. 6. Period-4 response. Top: time return maps for $U=3$ and $u_{\text {in }}=1.97$. Inset: $t_{n}$ versus $n$. Bottom: zero-contour of $\phi$ : (a) droplet \#59; (b) droplet \#60; (c) droplet \#61; and (d) droplet \#62. 


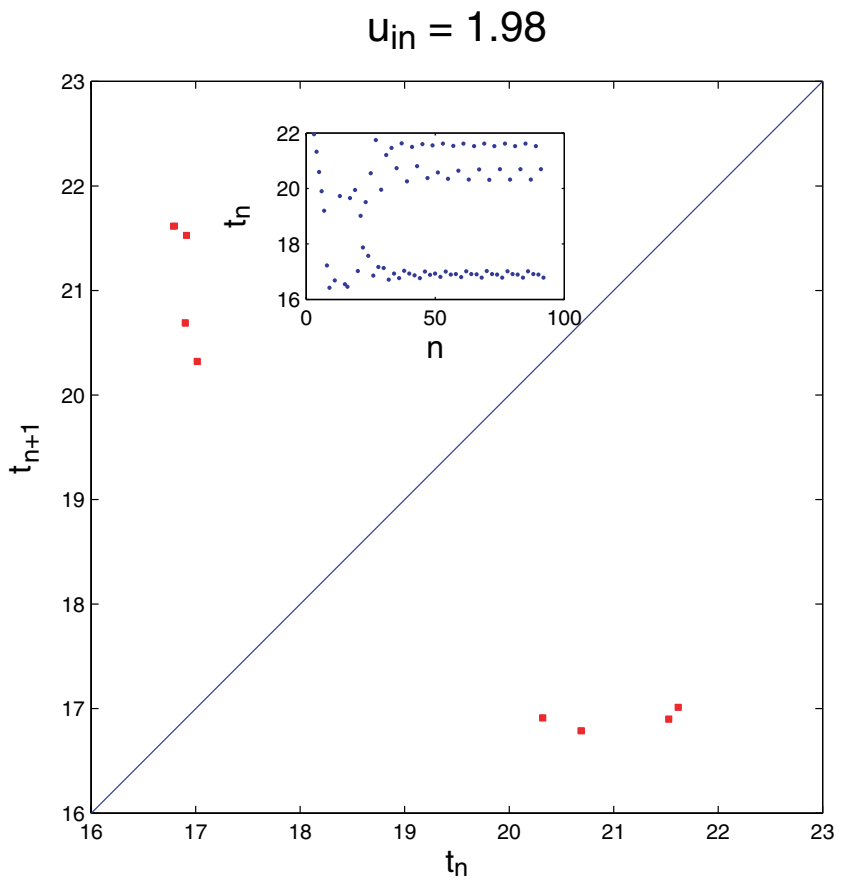

$$
u_{\text {in }}=2.1
$$

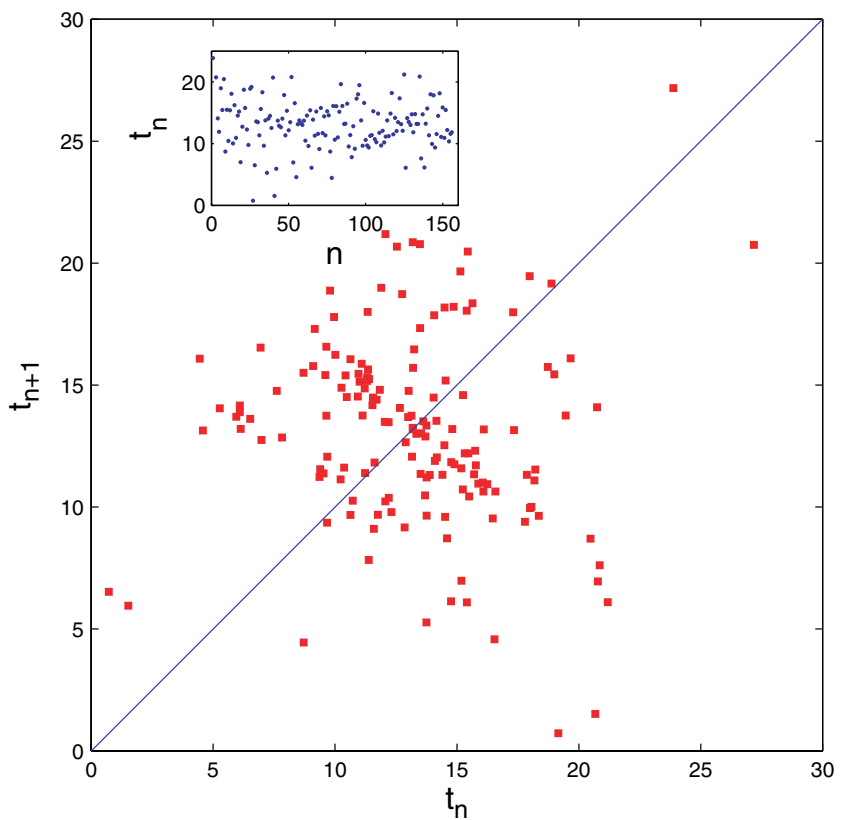

Fig. 7. Period-8 and chaotic responses. Top: time return map for $U=3$ and $u_{\text {in }}=1.98$. Inset: $t_{n}$ versus $n$. Bottom: time return maps for $U=3$ and $u_{\text {in }}=2.1$. Inset: $t_{n}$ versus $n$. 
Table 1. Summary of nonlinear dynamical results for the first class dripping-to-jetting transition. Here, the flow rate of outer fluid is fixed $(U=3)$.

\begin{tabular}{lccccccccc}
\hline$u_{\text {in }}$ & $1.5-1.945$ & $1.9475-1.96$ & 1.97 & 1.98 & 1.99 & 1.995 & $2-2.01$ & $2.015-2.05$ & $2.06-2.5$ \\
\hline Period & 1 & 2 & 4 & 8 & 16 & 8 & 4 & 2 & Chaotic \\
\hline
\end{tabular}

We are going to give a numerical investigation that the first class of drippingto-jetting transition is controlled by the Weber number of the inner fluid $\mathcal{W}_{\text {in }}=$ $\rho \bar{H}_{\text {orifice }} \bar{u}_{\text {in }}^{2} / \gamma$, and the second class of dripping-to-jetting transition is controlled by the capillary number of the outer fluid $\mathcal{C}_{\text {out }}=\eta \bar{U} / \gamma$. The following three cases of the first class of dripping-to-jetting transition are considered:

(1A) the case in Sec. 3.2;

(1B) increase the interfacial tension $\gamma$ in (1A) by $10 \%$; and

(1C) increase the density, $\rho$, in (1A) by $10 \%$.

The critical Weber number of the inner fluid is defined as $\mathcal{W}_{\mathrm{in}}^{*}=\rho \bar{H}_{\text {orifice }}\left(\bar{u}_{\mathrm{in}}^{*}\right)^{2} / \gamma$ where $\bar{u}_{\mathrm{in}}^{*}$ is the critical velocity of the inner fluid for the first class of dripping-tojetting transition. We are going to investigate by numerical experiments that the critical Weber number $\mathcal{W}_{\text {in }}^{*}$ in cases $(1 \mathrm{~A})-(1 \mathrm{C})$ are almost the same. For the second

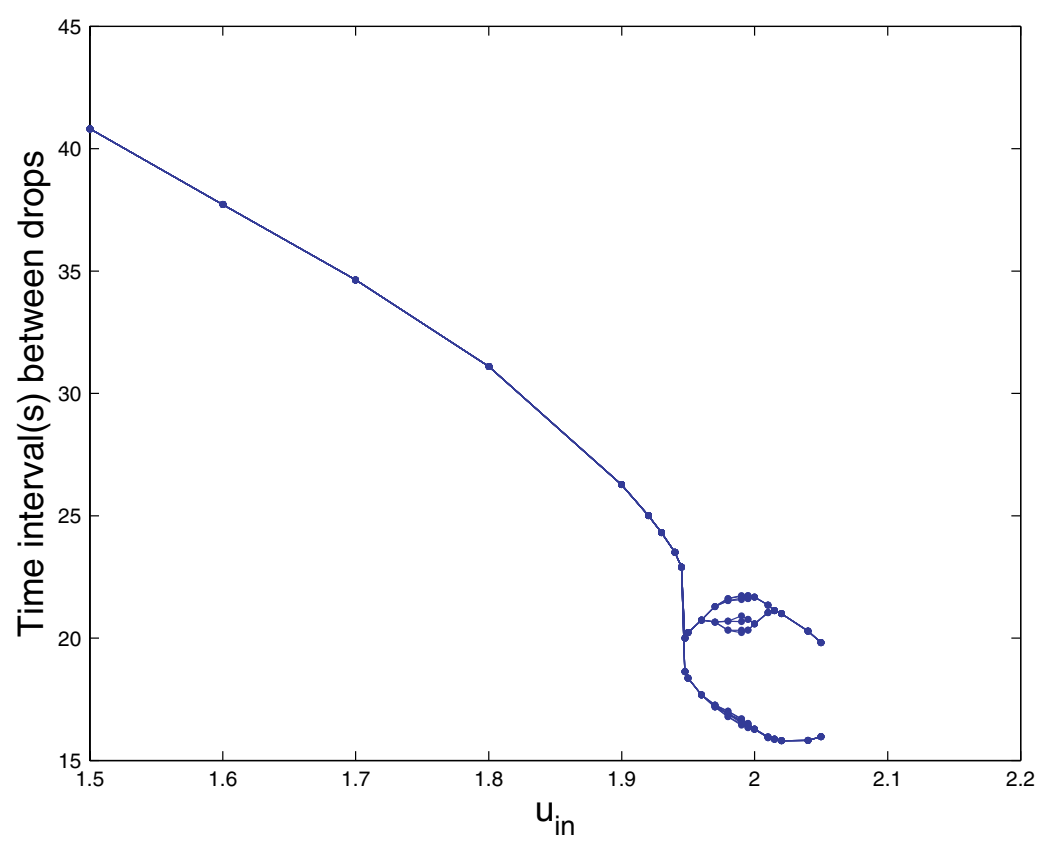

(a)

Fig. 8. Bifurcation diagram of pinch-off time for fixed $U=3$ and different $u_{\text {in }}$ (b) and (c) are two different close-ups of (a). 


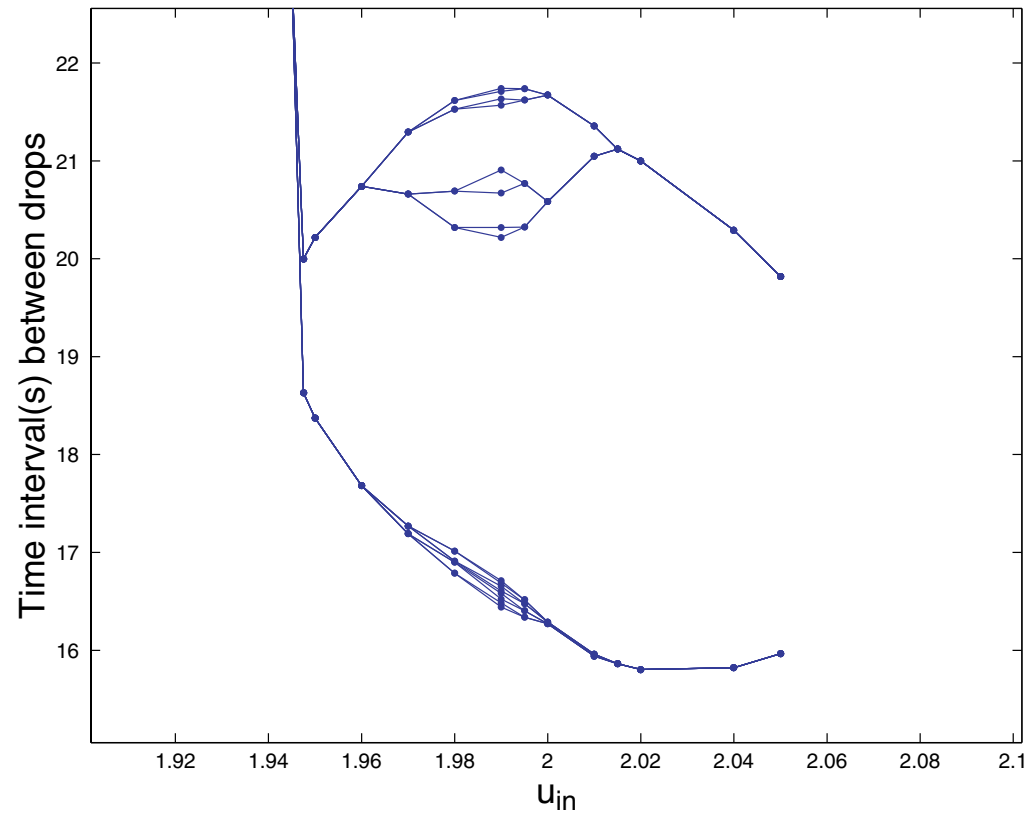

(b)

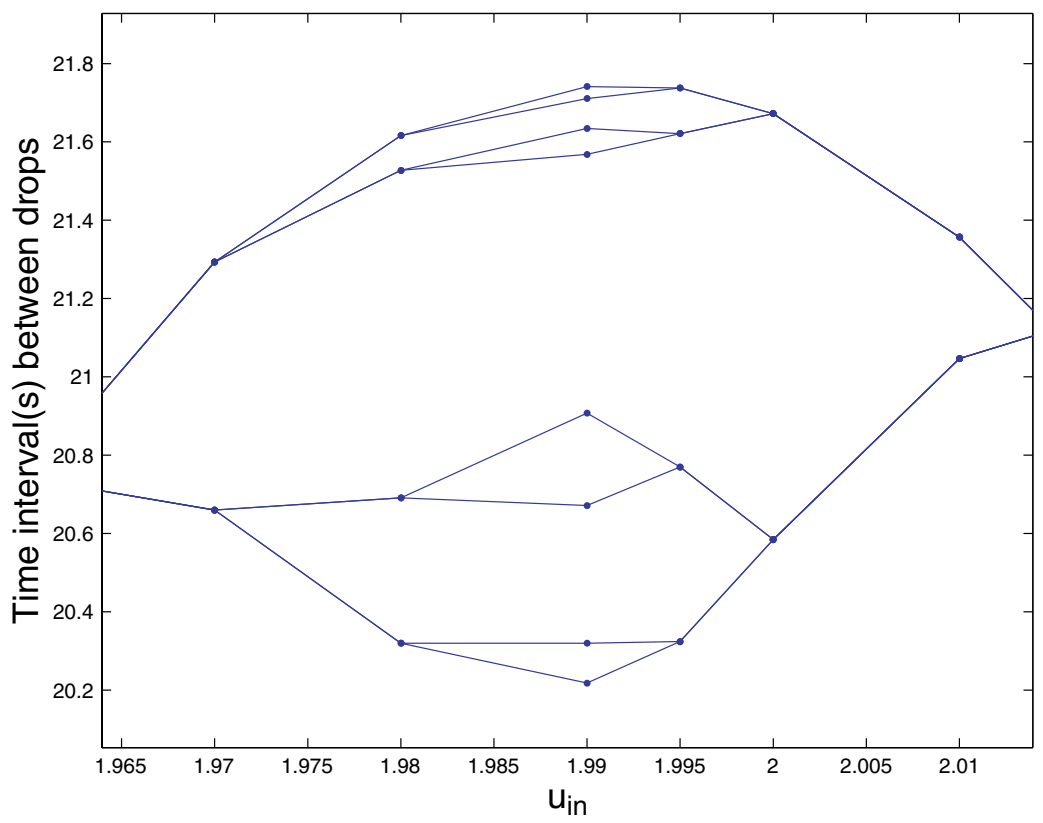

(c)

Fig. 8. (Continued) 
Table 2. Summary of nonlinear dynamical results for the second class dripping-to-jetting transition. Here, the flow rate of inner fluid is fixed $\left(u_{\mathrm{in}}=1.9\right)$.

\begin{tabular}{cccccc}
\hline$U$ & $3.05-3.19$ & $3.2-3.5$ & $3.55-3.65$ & $3.7-3.75$ & $3.8-4.3$ \\
\hline Period & 1 & Chaotic & 1 & Chaotic & 1 \\
\hline
\end{tabular}

class of dripping-to-jetting transition, the following three cases are considered:

(2A) the case in Sec. 3.2; (Therefore, (1A) and (2A) have the same set of controlling parameters.)

(2B) decrease the viscosity $\eta$ of (2A) by $10 \%$; and

$(2 \mathrm{C})$ increase the interfacial tension $\gamma$ of $(2 \mathrm{~A})$ by $10 \%$. (Note that $(2 \mathrm{C})$ and $(1 \mathrm{~B})$ are the same.)

The critical capillary number of the outer fluid is defined as $\mathcal{C}_{\text {out }}^{*}=\eta \bar{U}^{*} / \gamma$ where $\bar{U}^{*}$ characterizes the critical velocity of the outer fluid $u_{\text {out }}^{*}$ for the second class of dripping-to-jetting transition. We are going to investigate by numerical experiments that the critical capillary number of the outer fluid $\mathcal{C}_{\text {out }}^{*}$ in cases $(2 \mathrm{~A})-(2 \mathrm{C})$ are almost the same.

Now let us find those controlling parameters in the mathematical model for the above six different cases. First of all, the dimensionless parameters controlling the mathematical model for Cases (1A) and (2A) are given in Sec. 2:

$$
\begin{aligned}
& \mathcal{L}_{d}=M r / \xi V=5 \\
& \mathcal{R}=\rho \xi V / \eta=0.03 \\
& \mathcal{B}=r^{2} \xi / u \eta V=\frac{3}{2 \sqrt{2}} \gamma / \eta V=12 \\
& \mathcal{V}_{s}=K \Gamma / V=5
\end{aligned}
$$

Some basic parameters come from MD simulations of moving contact line problem [Qian et al. (2003)], and are listed in Table 3. For simplicity, we neglect their unit.

For the Case (1B), interfacial tension $\gamma$ is increased by $10 \%$, i.e., $\gamma=1.1(4.75)=$ 5.225. Then, by $\sqrt{r / u}=1$ and $\gamma=2 \sqrt{2} r^{2} \xi / 3 u$, we obtain $r=u=16.626$. From $\xi=$ $\sqrt{K / r}$, we get $K=1.8473$. From the definition of those dimensionless parameters controlling the mathematical model, we obtain $\mathcal{L}_{d}=5.5025, \mathcal{B}=13.1998, \mathcal{V}_{s}=$ 5.5014 , and $\mathcal{R}$ remains unchanged. For the Case (1C), density $\rho$ is increased by

Table 3. Basic parameters for Cases (1A) and (2A): $M$ and $\Gamma$ are two phenomenological parameters, $\xi$ and $V$ are length and velocity units in the continuum model respectively, $\rho$ is the density, $\gamma$ is the interfacial tension and $\eta$ is the viscosity. Parameters $r$ and $u$ are determined from the equations $\sqrt{r / u}=1$ and $\gamma=2 \sqrt{2} r^{2} \xi / 3 u$. Then, $K$ is obtained from the equation $\xi=\sqrt{K / r}$.

\begin{tabular}{lccccccccc}
\hline$M$ & $\Gamma$ & $\xi$ & $V$ & $\rho$ & $\gamma$ & $\eta$ & $r$ & $u$ & $K$ \\
\hline 0.02758 & 0.7445 & $1 / 3$ & 0.25 & 0.6046 & 4.75 & 1.6794 & 15.11 & 15.11 & 1.6789 \\
\hline
\end{tabular}


$10 \%$, i.e., $\rho=1.1(0.6046)=0.6651$. Then, only $\mathcal{R}$ is changed to 0.033 and the other three parameters remain unchanged. For the Case (2B), viscosity $\eta$ is decreased by $10 \%$, i.e., $\eta=0.9(1.6794)=1.5115$. Then, $\mathcal{R}=0.0333, \mathcal{B}=13.3333$, and the other parameters remain unchanged. The parameters for Case $(2 \mathrm{C})$ are the same as those of Case (1B). The parameters controlling the mathematical model for all the above cases are summarized in Table 4.

Limiting length is defined as the distance from the orifice to the tip of the droplet at the moment that it pinch-off. Figure 9 shows the limiting length of a droplet. For each velocity, the stationary limiting length is measured. Starting from dripping (small velocity) and increasing the velocity, when there is a jump in the limiting length, the critical velocity is determined.

Figures 10-12 show the graphs of limiting length $L$ versus $u_{\text {in }}$ with $U=3$ for Cases (1A)-(1C), respectively. Figures 13-15 show the graphs of limiting length

Table 4. Coefficients for PDEs (1)-(4) for all different cases.

\begin{tabular}{cllll}
\hline Case & \multicolumn{1}{c}{$\mathcal{L}_{d}$} & \multicolumn{1}{c}{$\mathcal{R}$} & \multicolumn{1}{c}{$\mathcal{B}$} & \multicolumn{1}{c}{$\mathcal{V}_{s}$} \\
\hline$(1 \mathrm{~A}),(2 \mathrm{~A})$ & 5 & 0.03 & 12 & 5 \\
$(1 \mathrm{~B}),(2 \mathrm{C})$ & 5.5025 & 0.03 & 13.1998 & 5.5014 \\
$(1 \mathrm{C})$ & 5 & 0.033 & 12 & 5 \\
$(2 \mathrm{~B})$ & 5 & 0.0333 & 13.3333 & 5 \\
\hline
\end{tabular}

\section{Limiting length $\mathrm{L}$}

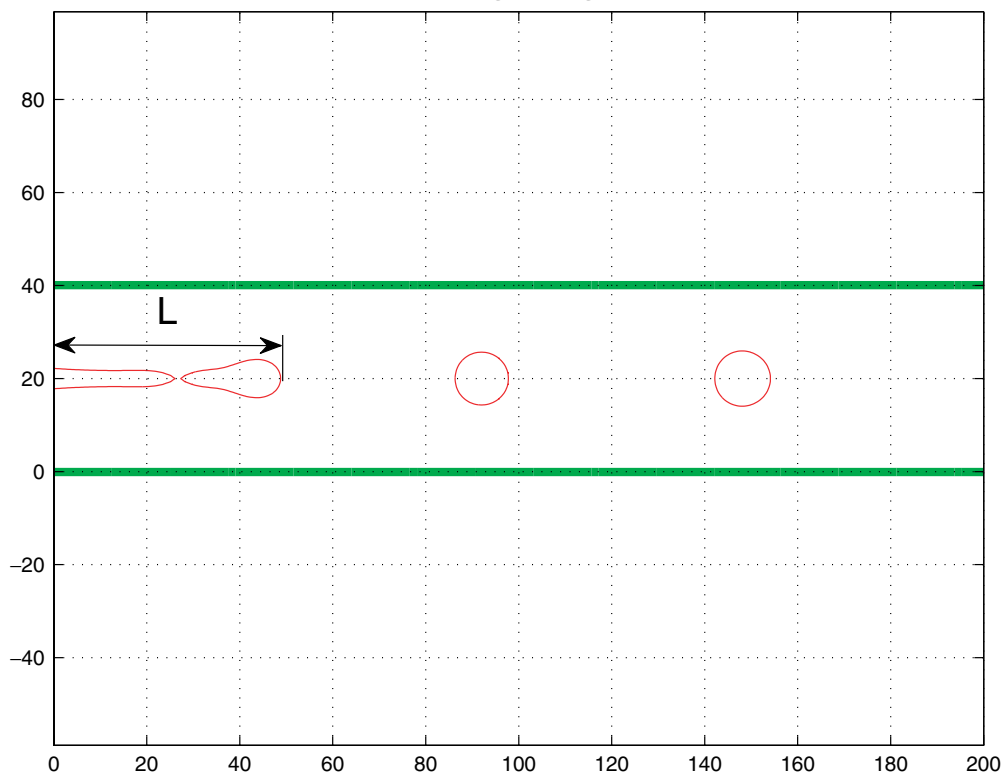

Fig. 9. Limiting length, $L$, of a droplet: the distance from the orifice to the tip of the droplet at pinch-off moment. 

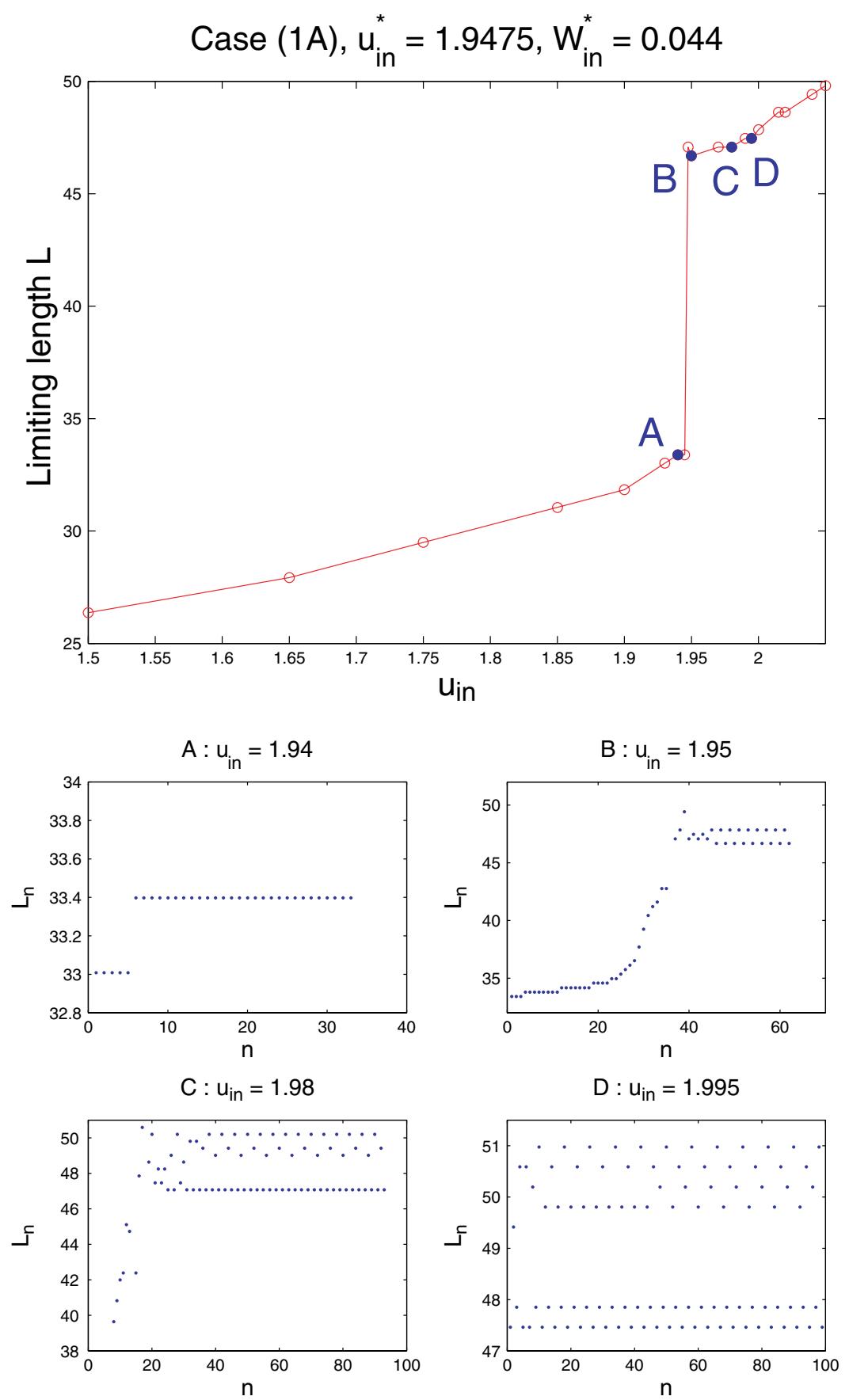

Fig. 10. Top: Limiting length $L$ versus $u_{\text {in }}$ for the case $(1 \mathrm{~A})$ - original parameter set. Bottom: the sequence of limiting length $L_{n}$ to the $n$th droplet for some fixed $u_{\text {in }}$. 

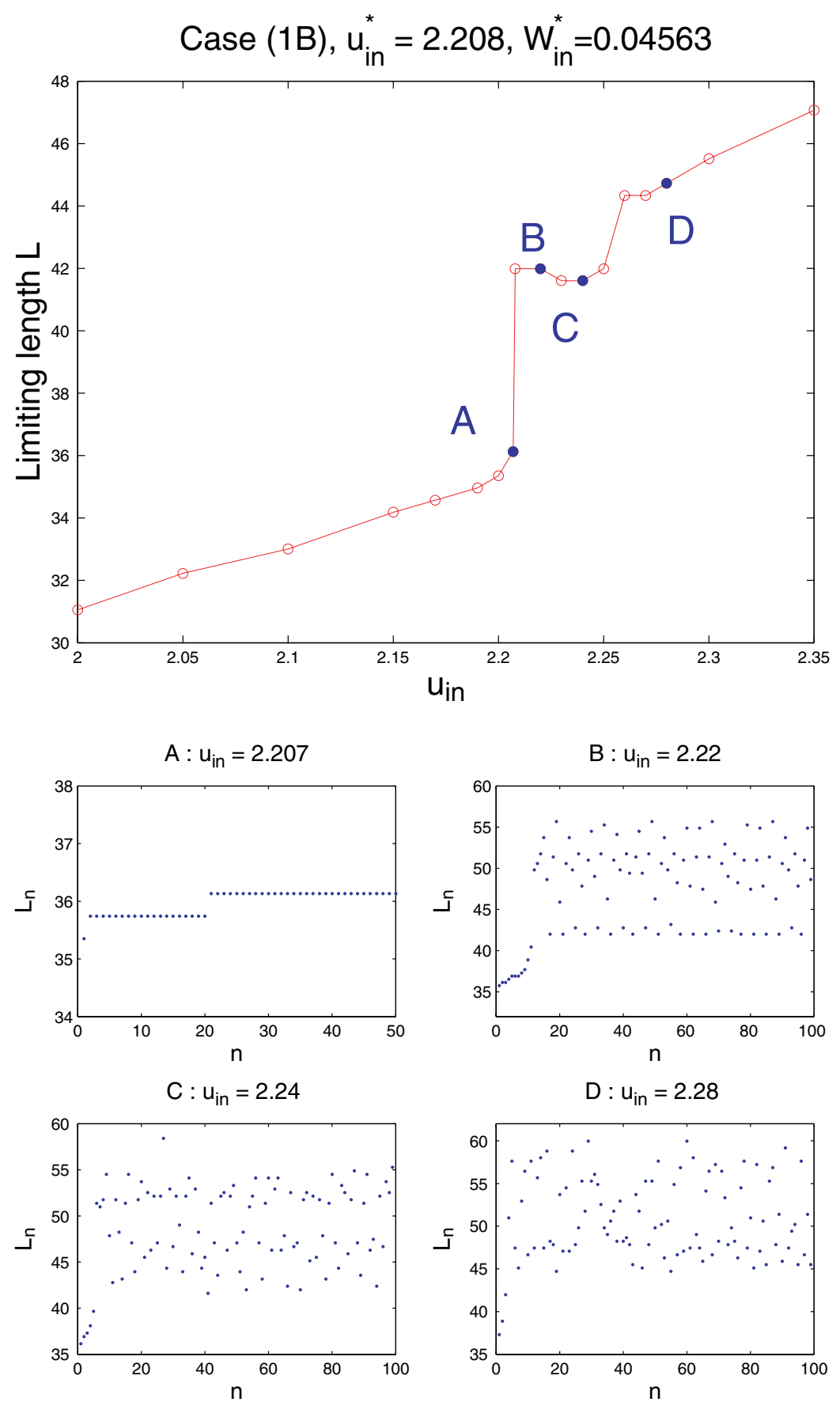

Fig. 11. Top: Limiting length $L$ versus $u_{\text {in }}$ for the case $(1 \mathrm{~B})-\gamma / 10 \%$. Bottom: the sequence of limiting length $L_{n}$ to the $n$th droplet for some fixed $u_{\text {in }}$. 

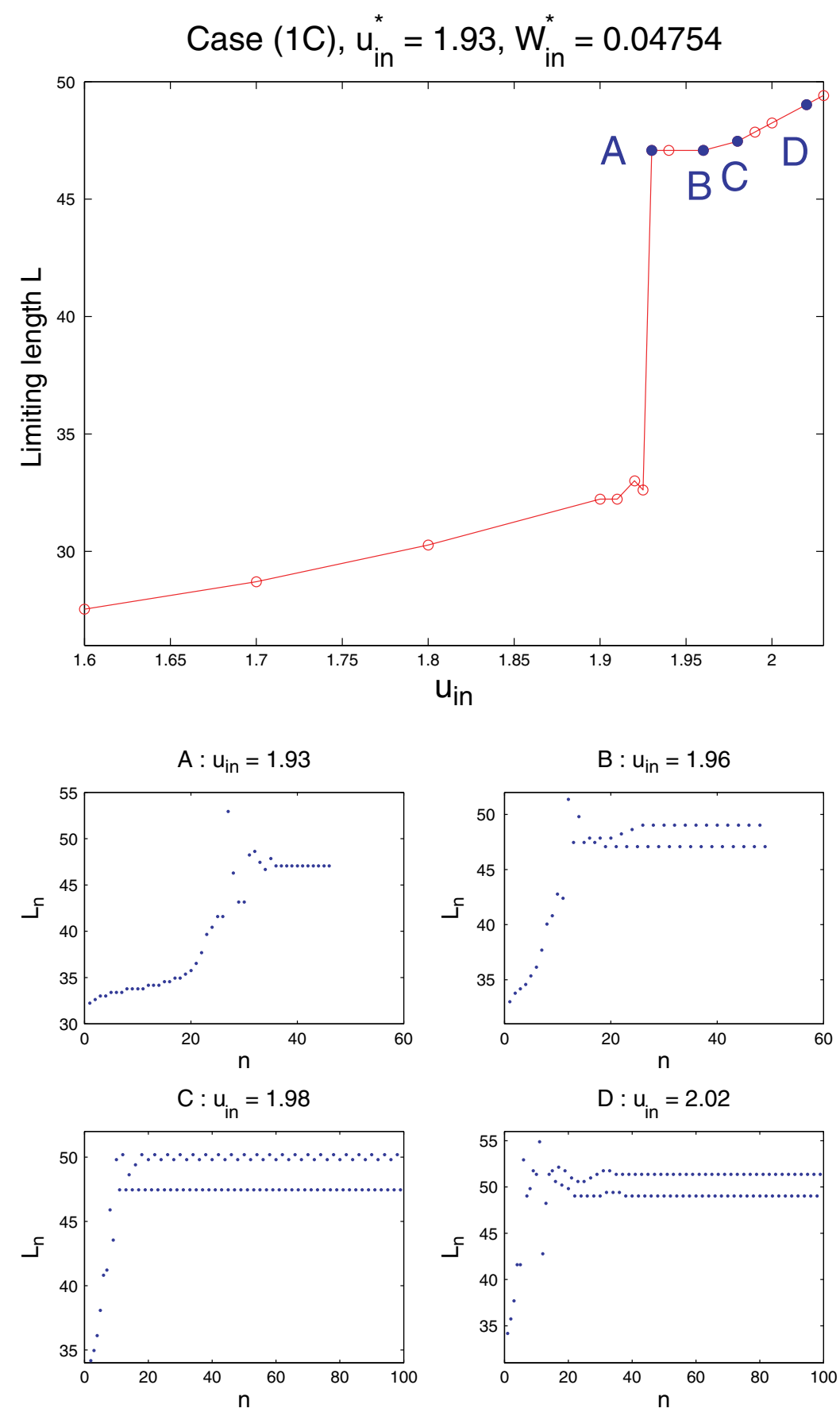

Fig. 12. Top: Limiting length $L$ versus $u_{\text {in }}$ for the case (1C) $-\rho \nearrow 10 \%$. Bottom: the sequence of limiting length $L_{n}$ to the $n$th droplet for some fixed $u_{\text {in }}$. 

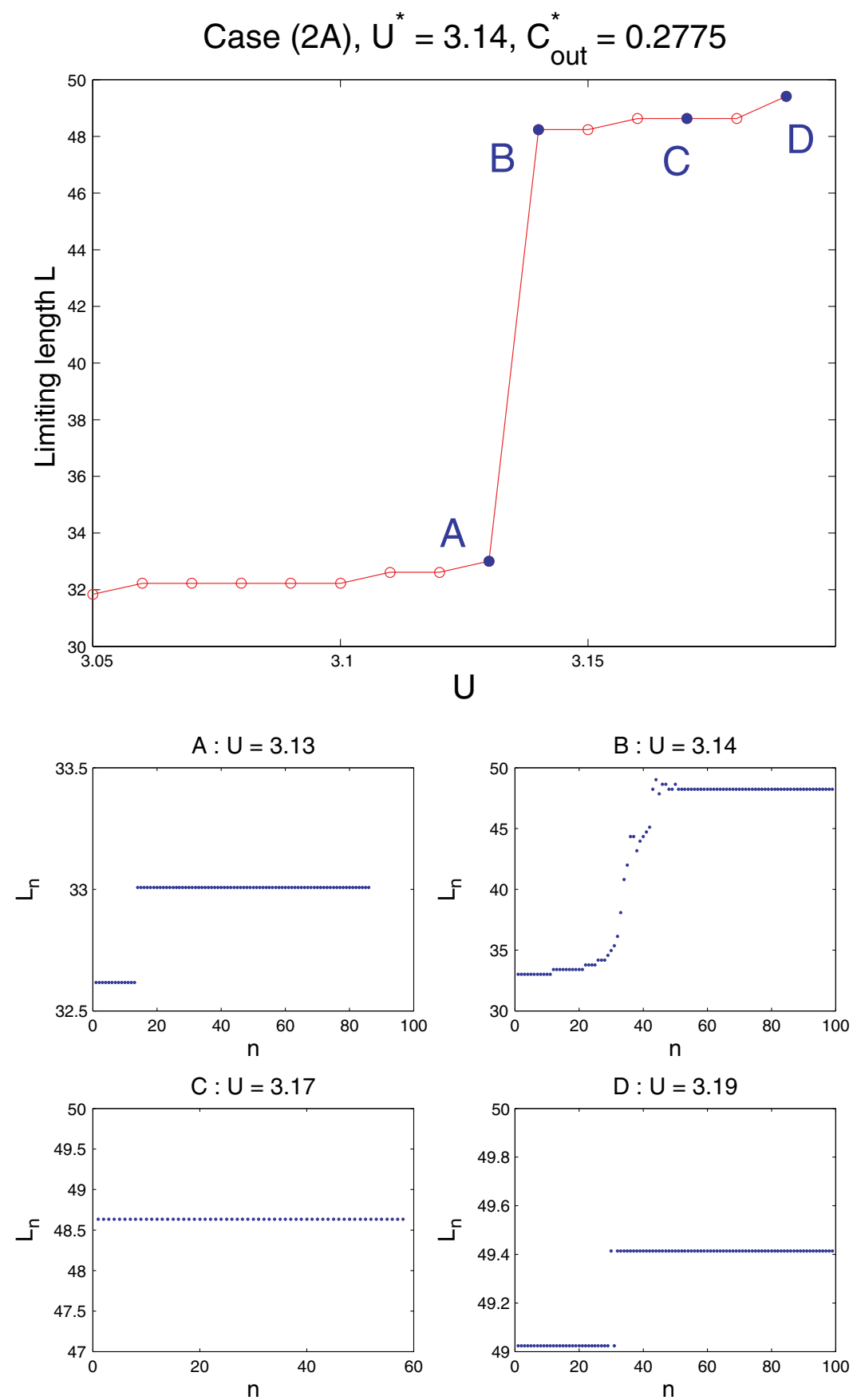

Fig. 13. Top: Limiting length $L$ versus $u_{\text {in }}$ for the case $(2 \mathrm{~A})$ - original parameter set. Bottom: the sequence of limiting length $L_{n}$ to the $n$th droplet for some fixed $U$. 

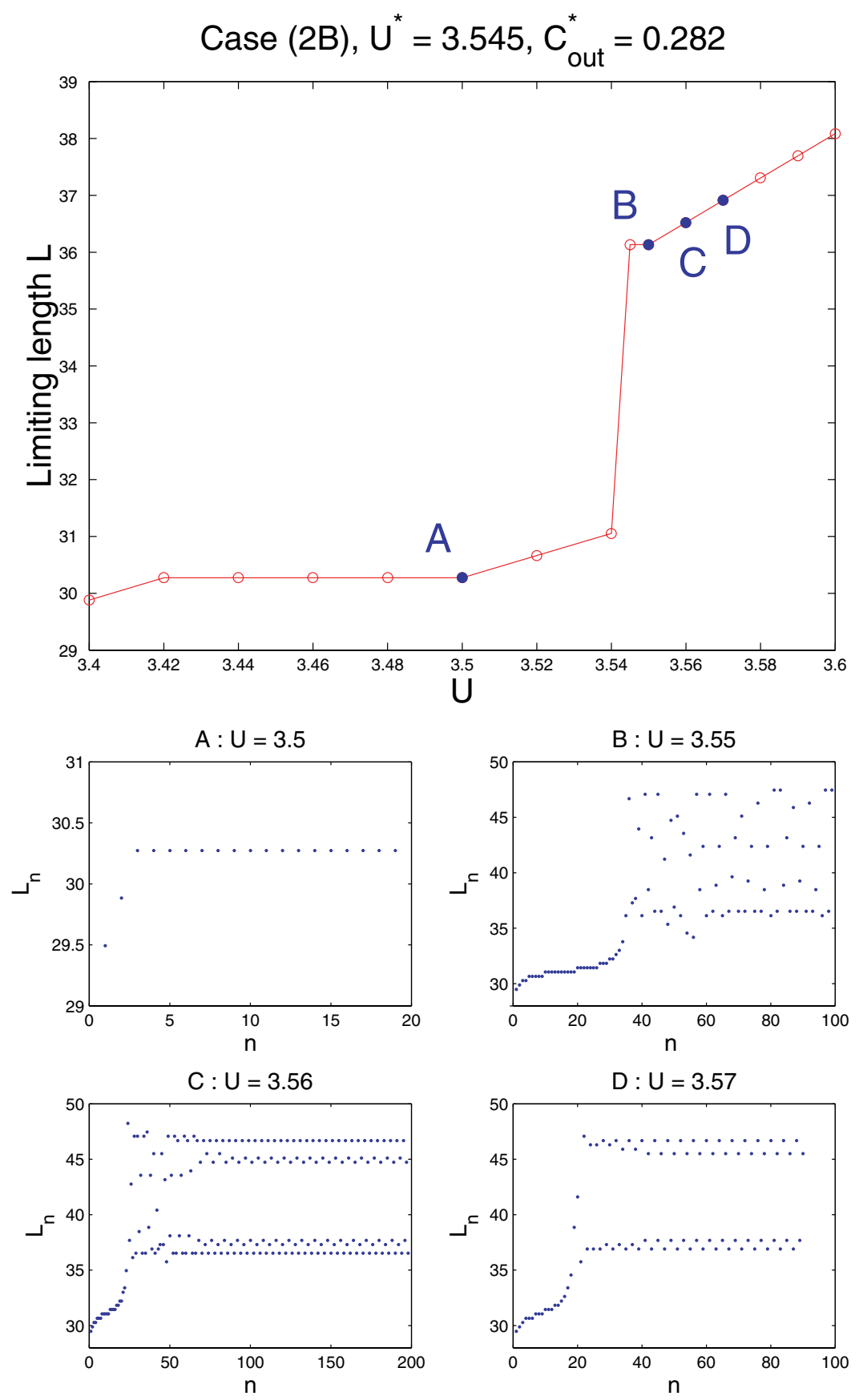

Fig. 14. Top: Limiting length $L$ versus $u_{\text {in }}$ for the case $(2 \mathrm{~B})-\eta \backslash 10 \%$. Bottom: the sequence of limiting length $L_{n}$ to the $n$th droplet for some fixed $U$. 

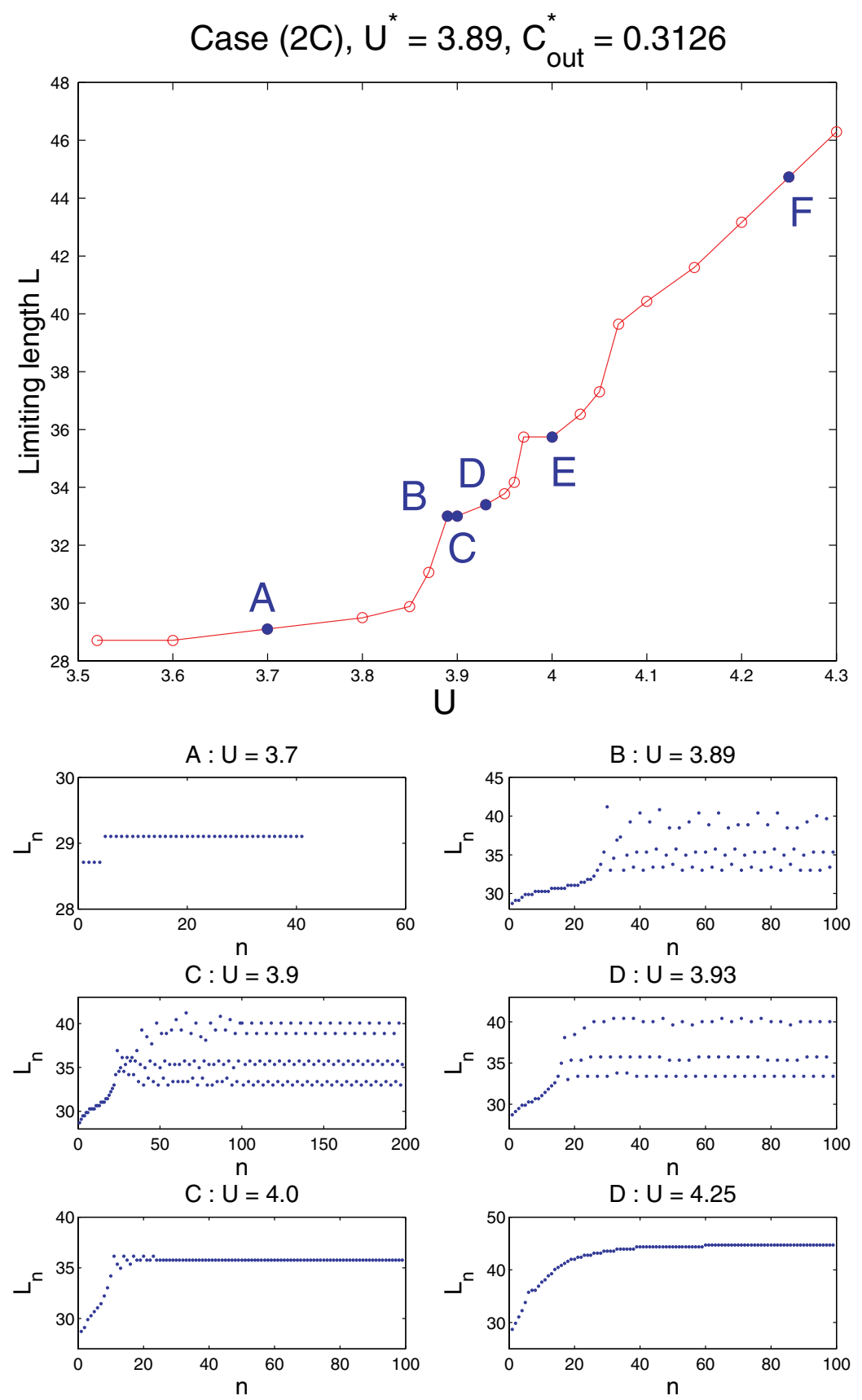

Fig. 15. Top: Limiting length $L$ versus $u_{\text {in }}$ for the case $(2 \mathrm{C})-\gamma / 10 \%$. Bottom: the sequence of limiting length $L_{n}$ to the $n$th droplet for some fixed $U$. 
Table 5. Critical Weber number $\mathcal{W}_{\text {in }}^{*}$ and capillary number $\mathcal{C}_{\text {out }}^{*}$ for all cases.

\begin{tabular}{llllllll}
\hline Case & $(1 \mathrm{~A})$ & $(1 \mathrm{~B})$ & \multicolumn{1}{c}{$(1 \mathrm{C})$} & Case & $(2 \mathrm{~A})$ & $(2 \mathrm{~B})$ & $(2 \mathrm{C})$ \\
\hline$u_{\text {in }}^{*}$ & 1.9475 & 2.208 & 1.93 & $U^{*}$ & 3.14 & 3.545 & 3.85 \\
$\mathcal{W}_{\text {in }}^{*}$ & 0.044 & 0.04563 & 0.04754 & $\mathcal{C}_{\text {out }}^{*}$ & 0.2775 & 0.282 & 0.3094 \\
\hline
\end{tabular}

$L$ versus $U$ with $u_{\text {in }}=1.9$ for cases $(2 \mathrm{~A})-(2 \mathrm{C})$, respectively. The insets of these figures show the sequence of limiting length $L_{n}$ of the $n$th droplet for fixed $u_{\text {in }}$ and $U$. Note that nonlinear dynamical phenomena are observed also for $\left\{L_{n}\right\}$. Therefore, the stationary limiting length is not unique. To determine the critical velocity of the dripping-to-jetting transition and plotting the graph $L$ versus velocity, we take the minimum stationary limiting length as $L$ for multiperiodic responses. For chaotic response, the "principle" minimum limiting length is taken.

Table 5 shows the critical velocities $u_{\mathrm{in}}^{*}$ and $U^{*}$, critical Weber numbers of the inner fluid $\mathcal{W}_{\text {in }}^{*}$ and critical capillary numbers of the outer fluid $\mathcal{C}_{\text {out }}^{*}$ for all cases. Note that, in the computation of $\mathcal{W}_{\mathrm{in}}^{*}$, we should use the dimensional values of $H_{\text {orifice }}$ and $u_{\text {in }}$, i.e., $\bar{H}_{\text {orifice }}=H_{\text {orifice }} \xi=4.375 / 3$ and $\bar{u}_{\text {in }}=u_{\text {in }} V=0.25 u_{\text {in }}$. Also, in the computation of $\mathcal{C}_{\text {out }}^{*}$, we need to use dimensional value of $U$, i.e., $\bar{U}=U V=0.25 U$.

From Table 5, it can be seen that the critical Weber number of the inner fluid $\mathcal{W}_{\text {in }}^{*}$ for Cases $(1 \mathrm{~A})-(1 \mathrm{C})$ are almost the same. This reflects that the first class of dripping-to-jetting transition is controlled by the Weber number of the inner fluid $\mathcal{W}_{\text {in }}=\rho \bar{H}_{\text {orifice }} \bar{u}_{\text {in }}^{2} / \gamma$. That is the balance between interfacial tension and inertial forces of the inner fluid. Besides, the critical capillary number of the outer fluid $\mathcal{C}_{\text {out }}^{*}$ for Cases $(2 \mathrm{~A})-(2 \mathrm{C})$ are almost the same. This reflects that the second class of dripping-to-jetting transition is controlled by the capillary number of the outer fluid $\mathcal{C}_{\text {out }}=\eta \bar{U} / \gamma$. That is the balance between interfacial tension and the viscous shear stresses on the droplet.

\section{Conclusion}

In this paper, we studied the dynamics of dripping-to-jetting transition for two immiscible coflowing liquid streams by solving the Cahn-Hilliard-Navier-Stokes system numerically. Two different classes of transition were identified. The first class is driven by the flow rate of the inner fluid $q_{\text {out }}$ and the second class is driven by the flow rate of the outer fluid $q_{\text {in }}$. In both cases, nonlinear dynamical phenomena such as period doubling and chaos were observed. Moreover, through extensive numerical calculations, it was shown that the first class of dripping-to-jetting transition is controlled by the Weber number of the inner fluid $\mathcal{W}_{\text {in }}$, and the second class of dripping-to-jetting transition is controlled by capillary number of the outer fluid $\mathcal{C}_{\text {out }}$. 


\section{Acknowledgments}

This research was based on work supported in part by award number SA-C0040/UK-C0016, made by King Abdullah University of Science and Technology (KAUST), Hong Kong RGC-CERG grants 603107 and 604209, the National Basic Research Program of China under the project of 2009CB623200.

\section{References}

Ambravaneswaran, B., Subramani, H. J., Phillips, S. D. and Basaran, O. A. (2004). Dripping-jetting transitions in a dripping faucet. Phys. Rev. Lett., 93: 034501.

Ambravaneswaran, B., Phillips, S. D. and Basaran, O. A. (2000). Theoretical analysis of a dripping faucet. Phys. Rev. Lett., 85: 5332.

Clanet, C. and Lasheras, J. C. (1999). Transition from dripping to jetting. J. Fluid Mech., 383: $307-326$.

Coullet, P., Mahadevan, L. and Riera, C. S. (2005). Hydrodynamical models for the chaotic dripping faucet. J. Fluid Mech., 526: 1-17.

Hohenberg, P. C. and Halperin, B. I. (1977). Theory of dynamic critical phenomena. Rev. Mod. Phys., 49(3): 435-479.

Anderson, D. M., McFadden, G. B. and Wheeler, A. A. (1998). A diffuse-interface methods in fluid mechanics. Rev. Fluid Mech., 30: 139-165.

Johnston, H. and Liu, J.-G. (2002). Finite difference schemes for incompressible flow based on local pressure boundary conditions. J. Comput. Phys., 180: 120-154.

Plateau, J. (1849). Statique expérimentale et théorique des liquides soumis aux seules forces moléculaires. Acad. Sci. Bruxelles Mem., 23: 5.

Qian, T. Z., Wang, X. P. and Sheng, P. (2003). Molecular scale contact line hydrodynamics of immiscible flows. Phys. Rev. E, 68: 016306.

Qian, T. Z., Wu, C. M., Lei, S. L., Wang, X. P. and Sheng, P. (2009). Modeling and simulations for molecular scale hydrodynamics of moving contact line in immiscible two-phase flows. J. Phys. Condens. Matter, 21: 464119.

Rayleigh, L. (1879). On the capillary phenomena of jets. Proc. R. Soc. Lond., 29: 71-79.

Shaw, R. (1984). The Dripping Faucet as a Model Chaotic System, Aerial, Santa Cruz, CA).

Utada, A. S., Fernandez-Nieves, A., Stone, H. A. and Weitz, D. A. (2007). Dripping to jetting transitions in coflowing liquid streams. Phys. Rev. Lett., 99: 094502.

Wang, X. P., Qian, T. Z. and Sheng, P. (2008). Moving contact line on chemically patterned surfaces. J. Fluid Mech., 605: 59-78.

Wu, C. M., Lei, S. L., Qian, T. Z. and Wang, X. P. (2010). Stick-slip motion of moving contact line on chemically patterned surfaces. Commun. Comput. Phys., 7: 403-422. 